\title{
Quantifying bile acid malabsorption helps predict response and tailor sequestrant therapy
}

\author{
Authors: Oluwafikunayo Orekoya, ${ }^{A}$ John McLaughlin, ${ }^{B}$ Eugenia Leitao, ${ }^{C}$ Wendy Johns, ${ }^{D}$ Simon Lal ${ }^{\mathrm{E}}$ and Peter Paine ${ }^{\mathrm{F}}$
}

\begin{abstract}
Although recognised as a cause of chronic diarrhoea for over forty years, diagnostic tests and treatments for bile acid malabsorption (BAM) remain controversial. Recent National Institute for Health and Care Excellence (NICE) guidelines highlighted the lack of evidence in the field, and called for further research. This retrospective study explores the BAM subtype and severity, the use and response to bile acid sequestrants (BAS) and the prevalence of abnormal colonic histology. 264 selenium-75-labelled homocholic acid conjugated taurine (SeHCAT)-tested patient records were reviewed and the severity and subtype of BAM, presence of colonic histopathology and response to BAS were recorded. $53 \%$ of patients tested had BAM, with type2 BAM in $45 \%$ of patients with presumed irritable bowel syndrome. Colonic histological abnormalities were similar overall between patients with $(29 \%)$ or without $(23 \%)$ BAM $(p=0.46)$ and between BAM subtypes, with no significant presence of inflammatory changes. $63 \%$ of patients with BAM had a successful BAS response which showed a trend to decreased response with reduced severity. Colestyramine was unsuccessful in $44 \%(38 / 87)$ and $45 \%$ of these (17/38) were related to medication intolerance, despite a positive SeHCAT. $47 \%$ (7/15) of colestyramine failures had a successful colesevelam response. No patient reported colesevelam intolerance. Quantifying severity of BAM appears to be useful in predicting BAS response. Colesevelam was better tolerated than colestyramine and showed some efficacy in colestyramine failures. Colestyramine failure should not be used to exclude BAM. Colonic histology is of no relevance.
\end{abstract}

KEYWORDS: Diarrhoea, bile acid malabsorption, bile acid sequestrant, SeHCAT, colestyramine, colesevelam

Authors: Afoundation trainee, Royal Bolton Hospital, Bolton, UK; Bprofessor of gastroenterology, Faculty of Medical and Human Sciences, University of Manchester, Manchester, UK, and Department of Gastroenterology, Salford Royal Foundation Trust, Salford, UK; Clinical scientist, Department of Nuclear Medicine, Salford Royal Foundation Trust, Salford, UK; D Consultant physicist, Department of Nuclear Medicine, Salford Royal Foundation Trust, Salford, UK; Egastroenterologist, Department of Gastroenterology, Salford Royal Foundation Trust, Salford, UK; F gastroenterologist, Department of Gastroenterology, Salford Royal Foundation Trust, Salford, UK

\section{Introduction}

Although bile acid malabsorption (BAM) has been recognised as a cause of chronic diarrhoea for over forty years, ${ }^{1}$ controversy surrounds its diagnosis and treatment. BAM is common, especially among patients presumed to have diarrhoeapredominant irritable bowel disease (IBS-D). ${ }^{2}$ It remains underdiagnosed and inadequately treated by generalists and gastroenterologists in the UK. ${ }^{3}$ Recent National Institute for Health and Care Excellence (NICE) guidelines highlighted the lack of evidence in the field, and called for further research. ${ }^{4}$

BAM is currently categorised according to aetiology into three subtypes. ${ }^{5}$ Type- 1 is due to terminal ileal disease or resection; type-2 (idiopathic or primary) is characterised by normal ileal structure and histology; type-3 groups miscellaneous causes, including gastric surgery, vagotomy, cholecystectomy, pancreatitis, coeliac disease and diabetes mellitus. ${ }^{5-11}$ In the UK alone, it has been estimated that as many as 500,000 people currently treated for IBS-D may instead have undiagnosed type-2 BAM. ${ }^{12}$ This high prevalence, adverse impact on quality of life and potential for effective treatment with bile acid sequestrants (BAS) suggests the need for more appropriate diagnostic strategies and optimal treatment.

Scanning with selenium-75-labelled homocholic acid conjugated taurine (SeHCAT) is the commonest method for diagnosing BAM. A non-invasive test, it uses scintigraphy to measure seven-day retention of orally administered SeHCAT, providing a validated indication of faecal bile acid excretion which can be quantified to indicate severity ${ }^{8,13-17}$ Measuring serum 7-alpha-hydroxy-4-cholestene-3-one or fibroblast growth factor 19 (FGF-19) levels are alternative tests but are currently only available for research purposes in the UK. ${ }^{18-24}$

Colestyramine, the most commonly used BAS believed to reduce symptoms, is poorly tolerated due to its unpleasant texture and side effects, including nausea, bloating, flatulence and abdominal pain. ${ }^{8,10,12,25}$ Consequently, over half of people started on colestyramine discontinue the medication within a year, often sooner. ${ }^{26}$ Failing to respond to it may imply lack of concordance not lack of efficacy, rendering 'trial by treatment' a poor diagnostic test. The BAS colesevelam is currently unlicensed for use in BAM, but advocated as an alternative. ${ }^{25,27,28}$ Compared with colestyramine, colesevelam has a 4-6 times stronger binding affinity to bile acids. ${ }^{28,29}$ It also has a lower side-effect profile and fewer clinical 
Table 1. Prevalence of BAM by subtype and severity.

\begin{tabular}{|c|c|c|c|c|c|}
\hline Underlying pathology & $\begin{array}{l}\text { BAM present } \\
<15 \%\end{array}$ & $\begin{array}{l}\text { BAM absent } \\
>15 \%\end{array}$ & $\begin{array}{l}\text { Severe } \\
<5 \%\end{array}$ & $\begin{array}{l}\text { Moderate } \\
5 \text { to }<10 \%\end{array}$ & $\begin{array}{l}\text { Mild } \\
10 \text { to }<15 \%\end{array}$ \\
\hline Ileal disease, including resections ( $n=56$ ) & $43(77)$ & $13(23)$ & $34(61)$ & $4(7)$ & $5(9)$ \\
\hline Idiopathic $(n=159)$ & $71(45)$ & $88(55)$ & $28(18)$ & $28(18)$ & $15(9)$ \\
\hline Miscellaneous pathology $(n=49)^{a}$ & $25(51)$ & $24(49)$ & $16(33)$ & $6(12)$ & $3(6)$ \\
\hline Total $(n=264)$ & $139(53)$ & $125(47)$ & $78(30)$ & $38(14)$ & $23(9)$ \\
\hline
\end{tabular}

Values are shown as the number of patients and the percentage in the brackets represents the proportion of patients, for that underlying pathology, with a SeHCAT value within a specified range ( $\%$ of $n)$. aMiscellaneous pathologies in which SeHCAT was performed $(n=49)$ included cholecystectomy $(n=37)$, diabetes treated with oral medications including metformin $(n=3)$, coeliac disease $(n=1)$, pancreatitis $(n=2)$, bacterial overgrowth $(n=2)$ and other gastrointestinal surgeries $(n=4)$. BAM $=$ bile acid malabsorption; SeHCAT $=$ selenium-75-labelled homocholic acid conjugated taurine.

interactions. ${ }^{30}$ Nonetheless, data on clinical efficacy are limited for both these agents. ${ }^{22}$

Lastly, previous studies have shown that BAM is evident in up to $43 \%$ of patients with microscopic colitis. ${ }^{8,31}$ The majority of patients investigated for diarrhoea undergo colonoscopy with biopsy. Although bile acid-induced colonic mucosal damage has previously been noted on histology in animal perfusion studies, a formal study of colonic histological findings in patients found to have BAM has not been undertaken. ${ }^{32,33}$ This retrospective study from a tertiary centre with high usage of SeHCAT, aimed to identify the clinical characteristics of patients with BAM, to determine current use and potential efficacy of BAS, and to explore findings from colonic histology in patients diagnosed by SeHCAT.

\section{Methods}

All patients with chronic diarrhoea, defined as three or more loose stools per day for at least four weeks, who underwent SeHCAT testing between April 2005 and January 2011 and subsequent follow up at Salford Royal Foundation Trust, were included for retrospective review.

SeHCAT was carried out in a standardised manner according to the manufacturer's guidelines (GE Healthcare, UK). Three hours after oral administration of a capsule containing $370 \mathrm{kBq}$ SeHCAT, baseline background-corrected counts were measured using a gamma camera. Seven-day retention was calculated as a percentage of the baseline value.

In line with previous studies, a seven-day retention value of $<15 \%$ was considered abnormal and severity was determined using previously defined thresholds. ${ }^{12,34,35}$ A value of $<5 \%$ was defined as severe, 5 to $<10 \%$ as moderate and 10 to $<15 \%$ as mild BAM. ${ }^{22}$

The use of fully integrated electronic patient records since 2003 facilitated data collection. Records were reviewed retrospectively; clinical details including age, sex, SeHCAT retention, presence of relevant diseases and colonic histology were collected. Patients with BAM were categorised according to subtype. Clinical response was recorded in patients offered colestyramine or colesevelam. The definition of a positive response was, of necessity, qualitative, based on whether the record reported reduced bowel movement frequency, stools becoming more solid or an improved quality of life at subsequent review, which ranged from one to three months.

Comparisons were made using simple proportions and statistical significance ascertained using two-tailed $\mathrm{p}$ values from Fisher's exact test.

\section{Results}

270 patients underwent SeHCAT testing; the electronic patient records at the trust meant only six patients were excluded due to a lack of key data. A total of $264(98 \%)$ patients were included in the study. $70 \%$ were female, the median age was 47 years (range 17-82). 139 (53\%) had a seven-day retention of $<15 \%$. Of these, $23(17 \%)$ were classed as mild, $38(27 \%)$ moderate and $78(56 \%)$ severe.

\section{Subtype BAM prevalence in SeHCAT-tested patients}

The proportion of patients with a SeHCAT value within a specific range for each subtype is shown in Table 1.

25 of the 56 patients with ileal disease had undergone a previous ileal resection. The majority of these $(23 / 25(92 \%))$ were found to have moderate-to-severe type-1 BAM compared with only 15 of the $31(48 \%)$ unresected patients ( $p=0.0005$, Fisher's exact test).

Type-2 BAM was identified in $45 \%$ of patients clinically presumed to have IBS-D ( $n=71 / 159)$, and was moderate to severe in 56/159 (35\%) patients. Type-3 BAM was found in $25 / 49$ patients $(51 \%)$ and was moderate to severe in $22(45 \%)$. Of note, $21 / 37$ (57\%) patients with previous cholecystectomy were found to have BAM.

\section{Overall response to BAS treatment}

A total of 92 patients were treated with BAS with followup data available. A successful response was reported in 58 $(63 \%)$ patients after treatment with BAS, of which 49 had colestyramine first line, 2 had colesevelam first line and 7 had colesevelam second line. Fig 1A shows the proportions of patients with successful and unsuccessful responses to BAS treatment for each subtype of BAM. The response to BAS therapies was not significantly lower in the heterogeneous type3 group ( $\mathrm{p}=0.37$ ). Response to BAS therapies (Fig 1B) decreased with reduced severity of BAM, although not reaching statistical significance when comparing response between patients with mild and severe BAM $(\mathrm{p}=0.25)$.

\section{Colestyramine treatment}

Of the 139 patients with BAM, 123 (88\%) were documented as given colestyramine, with subsequent follow-up information available for 87 patients. Although practice varied, generally patients were prescribed low-dose colestyramine (2-4 g daily) and advised to titrate the dose based on their response to the 

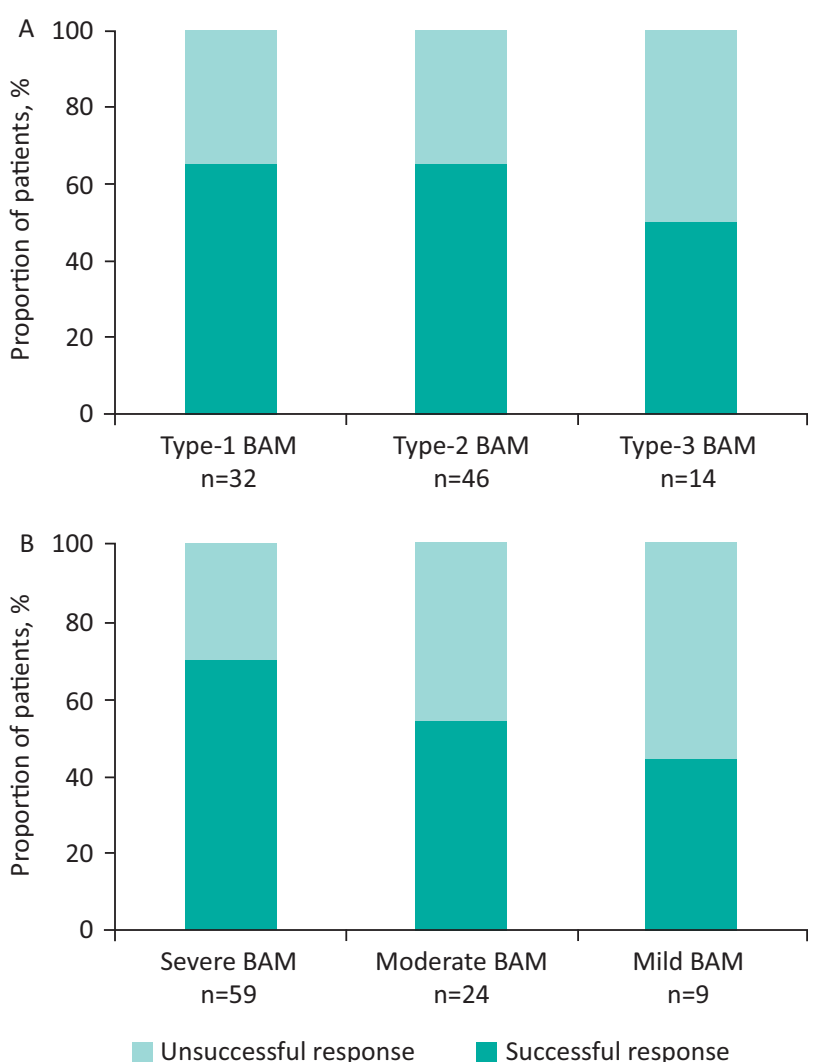

Fig 1. Proportion of patients with successful and unsuccessful responses to $B A S$ for each (A) subtype and (B) severity of BAM. BAM = bile acid malabsorption; $\mathrm{BAS}=$ bile acid sequestrants.

medication. The maximum dose ranged from 4-24 g (median $12 \mathrm{~g}$ ) but the duration of treatment was not reliably recorded. Table 2 shows the colestyramine treatment profiles of patients found to have BAM. Overall 38 patients with BAM (44\%) given colestyramine had an unsuccessful response, documented as being due to intolerance in 17 patients. This intolerance was due to unpalatability and side effects that included nausea, vomiting, flatulence and abdominal pain.

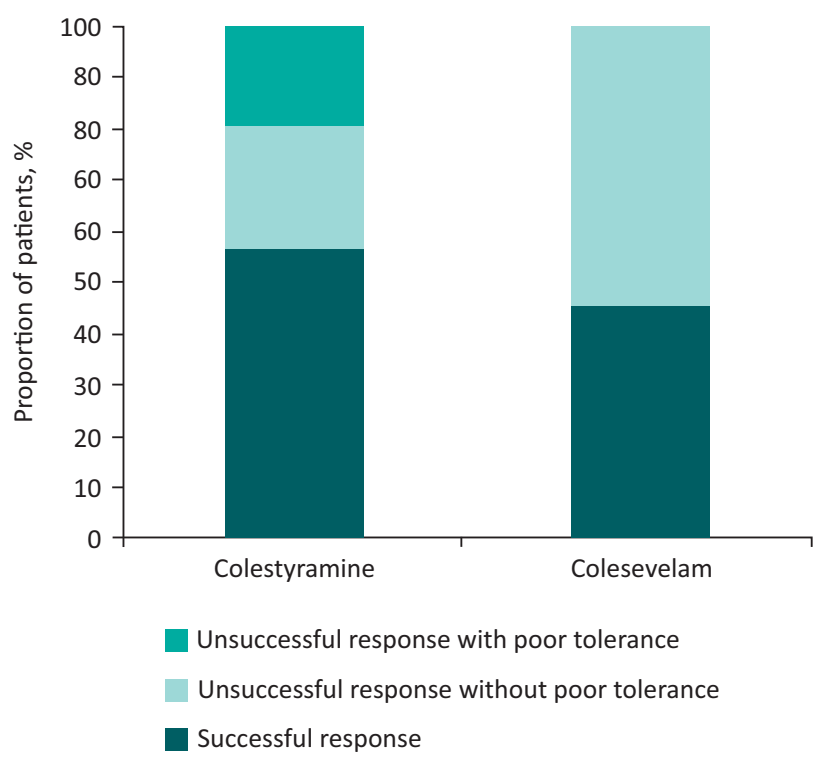

Fig 2. Overall response to colestyramine compared with response to first- or second-line colesevelam.

\section{Colesevelam treatment}

A successful response to first-line colesevelam was reported in $2 / 5(40 \%)$ patients given treatment. Colesevelam was offered as second-line BAS treatment more often to $45 \%$ of the colestyramine failures $(n=17 / 38)$. The dose of colesevelam first or second line ranged between 1.25 and $3.75 \mathrm{mg}$ per day. Follow-up information was available in 15 patients with $47 \%$ $(n=7 / 15)$ reporting a successful response. Failure to respond to colesevelam, first or second line, was never attributed to poor tolerance. Fig 2 compares overall response to colestyramine with response to first- or second-line colesevelam.

\section{Presence of histological abnormalities}

Clinically generated colonic histology reports were available for 150 patients tested with SeHCAT. There was no significant

\section{Table 2. Treatment profiles for the 139 patients offered colestyramine as first-line treatment.}

\begin{tabular}{|c|c|c|c|c|}
\hline Severity & Severe BAM $<5 \%$ & $\begin{array}{l}\text { Moderate BAM } \\
5 \text { to }<10 \%\end{array}$ & $\begin{array}{l}\text { Mild BAM } \\
10 \text { to }<15 \%\end{array}$ & $\begin{array}{l}\text { Total BAM } \\
<15 \%\end{array}$ \\
\hline Total patients, $\mathrm{n}$ & 78 & 38 & 23 & 139 \\
\hline Administered colestyramine, $\mathrm{n}$ & 72 & 36 & 15 & 123 \\
\hline Follow-up information available, $\mathrm{n}$ & 56 & 22 & 9 & 87 \\
\hline Successful response, $\mathrm{n}$ & $34(61)$ & $11(50)$ & $4(44)$ & $49(56)$ \\
\hline Unsuccessful response, $\mathrm{n}$ & 22 & 11 & 5 & 38 \\
\hline Ineffective & 12 & 6 & 3 & 21 \\
\hline Poor tolerance & 10 & 5 & 2 & 17 \\
\hline Given colesevelam, n (second-line) ${ }^{a}$ & 13 & 3 & 1 & 17 \\
\hline
\end{tabular}




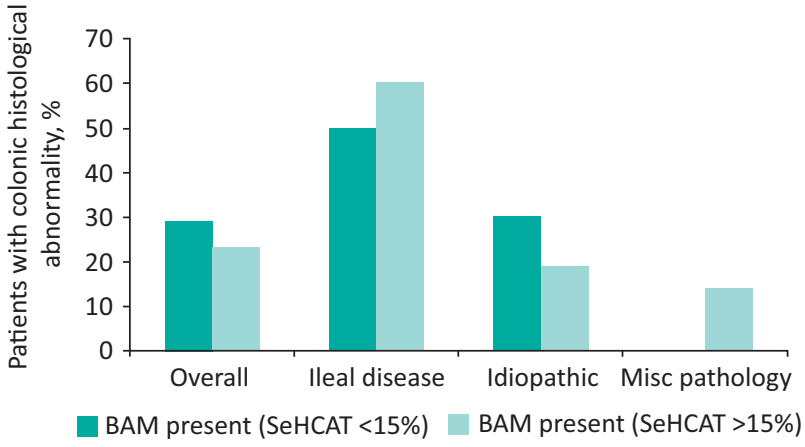

Fig 3. Presence of colonic histological abnormalities among patients with BAM compared with those without BAM. BAM = bile acid malabsorption.

difference in the presence of colonic histological abnormalities among patients with BAM $(29 \%, n=22 / 76)$ compared with those without BAM $(23 \%, \mathrm{n}=17 / 74)(\mathrm{p}=0.46)$, nor among the patients in the three subgroups (Fig 3; Table 3). In particular, of the range of histological abnormalities observed, there was no significant difference in inflammatory histological change between BAM-positive (16/76) and BAM-negative patients $(10 / 74)(\mathrm{p}=0.5)$.

Table 3. Proportion of patients with (SeHCAT <15\%) or without (SeHCAT >15\%) BAM found to have colonic histological abnormalities (patients with abnormality/total number of patients biopsied).

\begin{tabular}{llll} 
Disease & $\begin{array}{l}\text { SeHCAT } \\
<15 \%\end{array}$ & $\begin{array}{l}\text { SeHCAT } \\
>15 \%\end{array}$ & p value \\
Ileal disease (type-1 BAM) & $9 / 18$ & $5 / 8$ & 0.68 \\
Idiopathic (type-2 BAM) & $13 / 44$ & $10 / 52$ & 0.34 \\
$\begin{array}{l}\text { Miscellaneous pathology } \\
\text { (type-3 BAM) }\end{array}$ & $0 / 14$ & $2 / 14$ & 0.48 \\
Total & $22 / 76$ & $17 / 74$ & 0.46 \\
$\quad$ Ulceration & 3 & 2 & \\
$\quad$ Mild inflammation & 11 & 5 & \\
$\quad$ Moderate inflammation & 3 & 3 & \\
$\quad$ Chronic inflammation & 2 & 2 & \\
Polyps & 3 & 4 & \\
Granulomatous & 0 & 1 & \\
\hline
\end{tabular}

aTwo-tailed $p$ value from Fisher's exact test. The descriptive terms (mild, moderate, chronic etc) used to note histological abnormalities observed in colonic biopsies were directly taken from the pathologist's reports of the biopsies. Therefore these data were qualitative and may have varied between clinicians. However, there was no significant difference in inflammatory histological change between BAM positive (16/76) and BAM negative patients $(10 / 74)(p=0.5)$. Our series included no cases of either collagenous or lymphocytic colitis. BAM = bile acid malabsorption; SeHCAT = selenium-75labelled homocholic acid conjugated taurine.

\section{Discussion}

The relative paucity of data on BAM was highlighted by the recent NICE appraisal of SeHCAT, which recommended further research in the area. ${ }^{4}$ This retrospective study may help shape the key research questions.

Our data support the clinical importance of using SeHCAT scanning over the current approach of a 'trial by treatment' with colestyramine. In keeping with NICE's findings, this would not extend to type-1 BAM since almost all tested subjects with ileal disease and resection are, predictably, positive. ${ }^{16} \mathrm{~A}$ trial of treatment is therefore reasonable here.

We showed that a significant proportion of patients with proven BAM who were administered colestyramine reported poor tolerance or side effects. Empirically offering this agent without a diagnostic justification or driver is highly unlikely to prove effective. By utilising the SeHCAT test, clinicians can therefore avoid unnecessary trials of unpalatable colestyramine in patients without BAM, or falling into a trap of relying on false-negative 'colestyramine trials' in patients with BAM who have no motivating diagnosis and promptly stop. Such individuals who do have BAM are arguably less likely to then be offered colesevelam if a failed trial of colestyramine is taken as adequate to exclude BAM, a common misconception among practitioners which our data contests. Positive SeHCAT testing would bring the added knowledge of a definitive diagnosis of BAM and this would therefore encourage colestyramine continuation, tailoring of colestyramine dosage or switching to colesevelam if intolerant. However, a definitive prospective study is required.

Our data highlight that the potential benefit of colesevelam in people with BAM may be underutilised as only $45 \%$ of colestyramine non-responders were offered colesevelam as second-line treatment. Although our data suggests similar efficacy between first-line colesevelam and colestyramine and a lower side-effect profile with colesevelam, this observation is limited by the small number of patients given colesevelam first line $(n=5)$. Similarly, we cannot exclude a higher likelihood of placebo responsiveness, for example there may be greater initial expectation associated with second-line colesevelam. The small number of patients treated $(n=15)$ limits conclusions that can be drawn from this data. However, despite our small sample, our results agree with previous studies which reported symptom improvement and a lower incidence of side effects compared with colestyramine in patients with BAM taking colesevelam. ${ }^{25,28,36}$ A prospective and adequately powered study comparing both medicines in patients with BAM is therefore now warranted.

Utilising SeHCAT scanning would also guide clinical expectations of limited response in patients with mildto-moderate BAM. Our study showed that response to colestyramine (and combined BAS therapies) decreased with reduced severity of BAM, which concurs with a systematic review, specifically evaluating response in patients with type-2 BAM. ${ }^{12}$ However, given the small number of patients with mild BAM treated with BAS and followed up $(n=9)$, the relative efficacy of BAS cannot be fully determined. Lastly, scanning low-risk patients would encourage further investigation for another explanation for chronic diarrhoea in patients with SeHCAT retention $>15 \%$. Taken together with a negative faecal calprotectin and coeliac serology, an argument could be to place SeHCAT higher up the diagnostic algorithm for chronic diarrhoea in the absence of alarm features. 
This study, however, still advocates that an empirical trial of BAS in post-ileal resection diarrhoea may be warranted without SeHCAT testing, as nearly all patients with ileal resections had moderate-to-severe type-1 BAM, with many responding well to BAS treatment. This finding is in line with previous reports that found BAM in $90-100 \%$ of resected cases, which can be expected since the terminal ileum is the site for bile salt absorption..$^{2,8,16,37}$ On the other hand, as significantly fewer 'unresected' patients had moderate or severe disease, SeHCAT testing is likely to be more clinically useful in tailoring treatment among patients with unresected ileal disease.

Almost half of our patients, who would otherwise be labelled as patients with IBS-D, were found to have type-2 BAM. Similar to the findings of a systematic review, approximately onethird of these had moderate-to-severe BAM. ${ }^{12}$ Such evidence suggests that SeHCAT scanning is likely to be a clinically useful diagnostic tool for this group of patients as it will identify those more likely to respond to BAS treatment.

We found that colonic histological abnormalities are nonspecific and coincidental to the presence of BAM in patients with chronic diarrhoea, suggesting that the mechanism of diarrhoea is non-inflammatory. No cases of overt microscopic (collagenous/lympocytic) colitis were present in our series. Although our data show a marginally higher proportion of lower grade microscopic inflammation among patients with positive SeHCAT tests, the data collected were qualitative as they were based on narrative and descriptive terms in clinical pathology reports; the small differences were not significant. To explore this further, prospective quantitative studies are needed to assess the degree of inflammation or infiltration, by precise quantification of the number and types of inflammatory cells present in the colonic mucosa, but are beyond the scope of the current retrospective study. In view of these data, future studies are warranted.

Given that many patients thought to have IBS-D may have BAM, the scale and prevalence of the problem is likely to be large. A prospective, double-blind, placebo-controlled trial to compare the clinical and cost efficacy of SeHCAT and BAS is now needed. In particular, a larger study of first-line colesevalam would be timely, assessing its clinical and cost efficacy compared with SeHCAT retention. The field may also change if new diagnostics, such as serum $\mathrm{C} 4$, serum FGF-19, faecal bile acids or a combination, prove effective and accurate, possibly supplanting the need for SehCAT. 17,18,22,24 Nonetheless, making a positive diagnosis should be the clinician's goal in managing this unpleasant and often overlooked, yet treatable, condition.

\section{References}

1 Hofmann AF. The syndrome of ileal disease and the broken enterohepatic circulation: cholerhetic enteropathy. Gastroenterology 1967;52:752-7.

2 Smith MJ, Cherian P, Raju GS. Bile acid malabsorption in persistent diarrhoea. J R Coll Physicians Lond 2000;34:448-51.

3 Khalid U, Lalji A, Stafferton R, Andreyev J. Bile acid malabsoption: a forgotten diagnosis? Clin Med 2010;10:124-6.

4 National Institute for Health and Care Excellence. Diagnostics consultation document: SeHCAT (tauroselcholic [75selenium] acid) for the investigation of diarrhoea due to bile acid malabsorption. London:
NICE, 2012. Available online at www.nice.org.uk/guidance/dg7/ resources/sehcat-tauroselcholic-75selenium-acid-for-the-investigation-of-bile-acid-malabsorption-bam-and-measurement-of-bile-acidpool-loss-diagnostic-consultation-document2 [4 March 2015].

5 Fromm H, Malavolti M. Bile acid-induced diarrhoea. Clin Gastroenterol 1986;15:567-82.

6 Nyhlin H, Merrick MV, Eastwood MA. Bile acid malabsorption in Crohn's disease and indications for its assessment using SeHCAT. Gut 1994;35:90-3.

7 Williams AJ, Merrick MV, Eastwood MA. Idiopathic bile acid malabsorption - a review of clinical presentation, diagnosis, and response to treatment. Gut 1991;32:1004-6.

8 Wildt S, Nørby Rasmussen S, Lysgård Madsen J, Rumessen JJ. Bile acid malabsorption in patients with chronic diarrhoea: clinical value of SeHCAT test. Scand J Gastroenterol 2003;38:826-30.

9 al-Hadrani A, Lavelle-Jones M, Kennedy N. Bile acid malabsorption in patients with post-vagotomy diarrhoea. Ann Chir Gynaecol 1992;81:351-3.

10 Ford GA, Preece JD, Davies IH, Wilkinson SP. Use of the SeHCAT test in the investigation of diarrhoea. Postgrad Med J 1992;68:272-6.

11 Kurien M, Chaudhary S, McConnell JR. Letter: bile acid malabsorption - what is the prevalence in patients with chronic diarrhoea? Aliment Pharmacol Ther 2014;40:215.

12 Wedlake L, A'Hern R, Russell D. Systematic review: the prevalence of idiopathic bile acid malabsorption as diagnosed by SeHCAT scanning in patients with diarrhoea-predominant irritable bowel syndrome. Aliment Pharmacol Ther 2009;30:707-17.

13 Merrick MV, Eastwood MA, Anderson JR, Ross HM. Enterohepatic circulation in man of a gamma-emitting bile-acid conjugate, 23-selena-25-homotaurocholic acid (SeHCAT). J Nucl Med 1982;23:126-30.

14 Nyhlin H, Merrick MV, Eastwood MA, Brydon WG. Evaluation of ileal function using 23-selena-25-homotaurocholate, a-gammalabeled conjugated bile acid. Initial clinical assessment. Gastroenterology 1983;84:63-8.

15 Merrick MV, Eastwood MA, Ford MJ. Is bile acid malabsorption underdiagnosed? An evaluation of accuracy of diagnosis by measurement of SeHCAT retention. Br Med J (Clin Res Ed) 1985;290:665-8.

16 Gracie DJ, Kane JS, Mumtaz S et al. Prevalence of, and predictors of, bile acid malabsorption in outpatients with chronic diarrhea. Neurogastroenterol Motil 2012;24:983-e538.

17 Vijayvargiya P, Camilleri M, Shin A, Saenger A. Methods for diagnosis of bile acid malabsorption in clinical practice. Clin Gastroenterol Hepatol 2013;11:1232-9.

18 Walters JR, Tasleem AM, Omer OS et al. A new mechanism for bile acid diarrhea: defective feedback inhibition of bile acid biosynthesis. Clin Gastroenterol Hepatol 2009;7:1189-94.

19 Hofmann AF, Mangelsdorf DJ, Kliewer SA. Chronic diarrhea due to excessive bile acid synthesis and not defective ileal transport: a new syndrome of defective fibroblast growth factor 19 release. Clin Gastroenterol Hepatol 2009;7:1151-4.

20 Brydon WG, Nyhlin H, Eastwood MA, Merrick MV. Serum 7 alphahydroxy-4-cholesten-3-one and selenohomocholyltaurine (SeHCAT) whole body retention in the assessment of bile acid induced diarrhoea. Eur J Gastroenterol Hepatol 1996;8:117-23.

21 Pattni S, Walters JR. Recent advances in the understanding of bile acid malabsorption. Br Med Bull 2009;92:79-93.

22 Walters JR. Bile acid diarrhoea and FGF19: new views on diagnosis, pathogenesis and therapy. Nat Rev Gastroenterol Hepatol 2014;11:426-34

23 Pattni SS, Brydon WG, Dew T et al. Fibroblast growth factor 19 in patients with bile acid diarrhoea: a prospective comparison of FGF19 serum assay and SeHCAT retention. Aliment Pharmacol Ther 2013;38:967-76. 
24 Pattni SS, Brydon WG, Dew T, Walters JR. Fibroblast growth factor 19 and $7 \alpha$-hydroxy-4-cholesten-3-one in the diagnosis of patients with possible bile acid diarrhea. Clin Transl Gastroenterol 2012;3:e18.

25 Wilcox C, Turner J, Green J. Systematic review: the management of chronic diarrhoea due to bile acid malabsorption. Aliment Pharmacol Ther 2014;39:923-39.

26 Hiatt JG, Shamsie SG, Schectman G. Discontinuation rates of cholesterol-lowering medications: implications for primary care. Am J Manag Care 1999;5:437-44.

27 Beigel F, Teich N, Howaldt S et al. Colesevelam for the treatment of bile acid malabsorption-associated diarrhea in patients with Crohn's disease: A randomized, double-blind, placebo-controlled study. J Crohns Colitis 2014; 8:1471-9.

28 Wedlake L, Thomas K, Lalji A et al. Effectiveness and tolerability of colesevelam hydrochloride for bile-acid malabsorption in patients with cancer: a retrospective chart review and patient questionnaire. Clin Ther 2009 31:2549-58.

29 Steinmetz KL. Colesevelam hydrochloride. Am J Health Syst Pharm 2002;59:932-9.

30 Puleston J, Morgan H, Andreyev J. New treatment for bile salt malabsorption. Gut 2005;54:441-2.

31 Fernandez-Bañares F, Esteve M, Salas A et al. Bile acid malabsorption in microscopic colitis and in previously unexplained functional chronic diarrhea. Dig Dis Sci 2001;46:2231-8.
32 Camilleri M, Murphy R, Chadwick VS. Dose-related effects of chenodeoxycholic acid in the rabbit colon. Dig Dis Sci 1980;25:433-8.

33 Chadwick VS, Gaginella TS, Carlson GL et al. Effect of molecular structure on bile acid-induced alterations in absorptive function, permeability, and morphology in the perfused rabbit colon. J Lab Clin Med 1979;94:661-74.

34 Borghede MK, Schlütter JM, Agnholt JS et al. Bile acid malabsorption investigated by selenium-75-homocholic acid taurine ((75) SeHCAT) scans: causes and treatment responses to cholestyramine in 298 patients with chronic watery diarrhoea. Eur J Intern Med 2011;22:e137-40.

35 Sinha L, Liston R, Testa HJ, Moriarty KJ. Idiopathic bile acid malabsorption: qualitative and quantitative clinical features and response to cholestyramine. Aliment Pharmacol Ther 1998;12:839-44.

36 Aldridge MA, Ito MK. Colesevelam hydrochloride: a novel bile acid-binding resin. Ann Pharmacother 2001;35:898-907.

37 Kurien M, Evans KE, Leeds JS et al. Bile acid malabsorption: an under-investigated differential diagnosis in patients presenting with diarrhea predominant irritable bowel syndrome type symptoms. Scand J Gastroenterol 2011;46:818-22.

Address for correspondence: Dr O Orekoya, Royal Bolton Hospital, Minerva Road, Farnworth BL4 0JR, UK. Email: fikki.orekoya@nhs.net
The Future Hospital Programme (FHP) aims to develop and implement the RCP's vision for the future of medical care in hospital and community settings. We are keen to showcase examples of best and innovative practice to improve patient care, and would very much like to hear more about your work.

We are asking people to share their stories to create, grow and connect a future hospital network. These stories of best and innovative practice and patient care may be featured on the RCP website as exemplars of FHP principles and recommendations in action.

To share your story

Visit: www.rcplondon.ac.uk/FH-tell-us-your-story

For further information

Visit: www.rcplondon.ac.uk/FHP

or contact

futurehospital@rcplondon.ac.uk 